\title{
Modeling and simulation of a nanoscale optical computing system
}

\author{
Jun Pang ${ }^{\mathrm{a}, *}$, Alvin R. Lebeck ${ }^{\mathrm{b}}$, Christopher Dwyer ${ }^{\mathrm{c}, \mathrm{d}}$ \\ a Department of Computer Science, Duke University, D339 LSRC Bldg., 308 Research Drive, Campus Box 90129, Durham, NC 27708-0129, United States \\ ${ }^{\mathrm{b}}$ Department of Computer Science, Duke University, D308 LSRC Bldg., 308 Research Drive, Campus Box 90129, Durham, NC 27708-0129, United States \\ ${ }^{\mathrm{c}}$ Department of Electrical and Computer Engineering, Duke University, 209B Hudson Hall, Box 90291, Durham, NC 27708, United States \\ ${ }^{\mathrm{d}}$ Department of Computer Science, Duke University, 209B Hudson Hall, Box 90291, Durham, NC 27708, United States
}

\section{H I G H L I G H T S}

- We propose a method to build computing systems with nanoscale optical devices.

- An Optical Logic Element (OLE) is proposed as a basic compute unit.

- We demonstrate optical coupling between optical fibers and chromophores.

- We build and validate a SPICE model for OLEs, and analyze various logic circuits.

- OLE power-delay product is $2.5 \times$ lower and area density is $100 \times$ higher than CMOS.

\section{A R T I C L E I N F O}

\section{Article history:}

Received 19 February 2013

Received in revised form

24 July 2013

Accepted 29 July 2013

Available online 7 August 2013

\section{Keywords:}

Optical computing

DNA self-assembly

SPICE

RET logic

Nanophotonics

Emerging technology

\begin{abstract}
A B S T R A C T
Optical nanoscale computing is one promising alternative to the CMOS process. In this paper we explore the application of Resonance Energy Transfer (RET) logic to common digital circuits. We propose an Optical Logic Element (OLE) as a basic unit from which larger systems can be built. An OLE is a layered structure that works similar to a lookup table but instead uses wavelength division multiplexing for its inputs and output. Waveguides provide a convenient mechanism to connect multiple OLEs into large circuits. We build a SPICE model from first principles for each component to estimate the timing and power behavior of the OLE system. We analyze various logic circuits and the simulation results show that the components are theoretically correct and that the models faithfully reproduce the fundamental phenomena; the powerdelay product of OLE systems is at least $2.5 \times$ less than the $14 \mathrm{~nm}$ CMOS technology with $100 \times$ better density.
\end{abstract}

(c) 2013 Elsevier Inc. All rights reserved.

\section{Introduction}

The well-established scaling challenges that CMOS faces as feature sizes approach tens of nanometers have motivated research into alternative technologies for more than a decade. However, despite advances in next-generation transistor designs and foundry processes, power efficiency and thermal budgets continue to dominate the performance envelope of current systems.

Among the disruptive technology candidates that might one day supplement CMOS, the self-assembly of DNA into nanoscale logic circuits is a promising scheme. DNA can form structures with molecular precision, i.e., sub-nanometer, over several square

\footnotetext{
* Corresponding author.

E-mail addresses: pangjun92@gmail.com, pangjun@cs.duke.edu (J. Pang), alvy@cs.duke.edu (A.R. Lebeck), dwyer@ece.duke.edu (C. Dwyer).
}

microns and with massive assembly parallelism, e.g., $>10^{15}$ structures, in only a few hours of processing. A remaining challenge is to pattern useful building blocks on the DNA nanostructures to compose larger systems.

Molecular-scale computational devices have been patterned on DNA self-assembled grids and can be used to create simple logic circuits and computing systems [25,27]. Further, a variety of logic functions have been demonstrated including simple logic gates, combinational and sequential gate arrays with molecular devices [28]. However, the physical integration of molecular logic gates into serial cascades remains a problem because they often use incompatible inputs and outputs to represent binary signals.

In this paper, we demonstrate a method of integrating small molecules called chromophores into Resonance Energy Transfer (RET) logic gates on DNA nanostructures to build computing systems interconnected by optical waveguides, e.g., optical fibers. Similar to wavelength division multiplexing (WDM) input and 

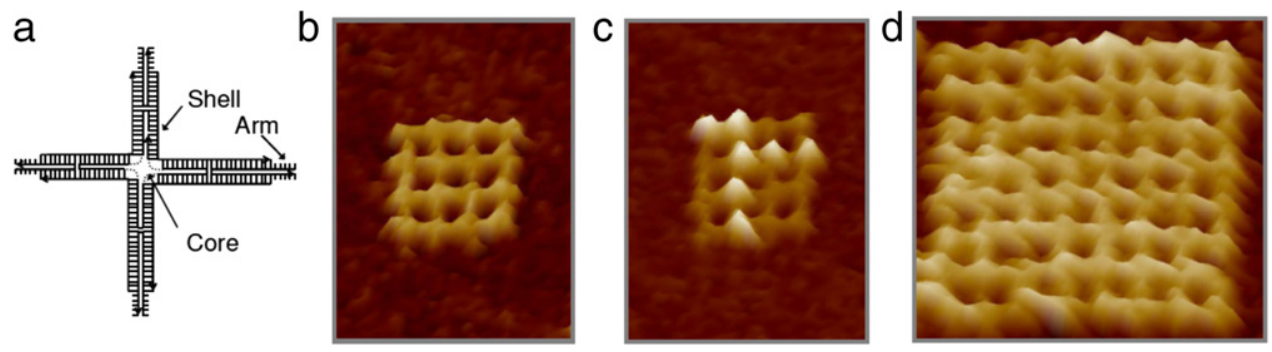

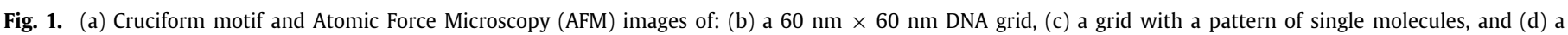
$140 \mathrm{~nm} \times 140 \mathrm{~nm}$ grid.

output signals in this system are represented by the presence (i.e., true) or absence (i.e., false) of a set of optical frequencies. We design an Optical Logic Element (OLE) as a basic unit from which larger systems can be built. An OLE is a microscale layered structure that works similar to a five-input lookup table (5-LUT) common in FPGAs, but instead uses wavelength division multiplexing for its inputs and output. An OLE has three basic components: (1) input and output color filters to select target wavelengths, (2) a nonlinear gain media, such as a layer of quantum dots, and (3) DNA self-assembled minterms implemented with RET logic. Optical fibers can be used as wires to interconnect OLEs into larger systems as a surrogate for microscale patterned waveguides currently used to create on-chip optical networks.

To demonstrate this scheme, we build a SPICE model from first principles for each component to estimate the timing and power behavior of an OLE and fiber. We analyze various logic circuits, e.g., a 2-input multiplexer, 1 bit full adder, an oscillator, a D-F/F, a carry-lookahead adder, etc. to understand their behaviors and design requirements for an experimental demonstration. Eventually, the SPICE model output will serve as a set of technology parameters for a larger-scale system simulation framework to evaluate architectural designs.

The simulation results show that the components are theoretically correct and that the models are self-consistent and faithfully reproduce the fundamental phenomena from which they were built. We also apply a power model for the OLE to show that the power-delay product (PDP) of those circuits is at least $2.5 \times$ less than that in state-of-art CMOS technology with at least two orders of magnitude better area density.

The rest of the paper is organized as follows: Section 2 describes DNA self-assembly and RET logic circuits. Section 3 introduces our system for optical computing including the OLE structure. Section 4 presents the details of the SPICE model for OLEs. Simulation results and analysis for a set of common logic blocks are shown in Section 5 and we conclude in Section 6.

\section{Background and motivation}

The OLE is the basic building block for the proposed system and is built from components that can be fabricated by conventional lithographic techniques with the exception of the logic minterms which we synthesize by DNA self-assembly. We have previously shown how DNA self-assembly can be used to build ultra-dense optical logic gates, e.g., $2 \mathrm{~nm} \times 2 \mathrm{~nm} \times 2 \mathrm{~nm}$ two-input AND gates [27], however, these structures are not easily interconnected into larger systems. In order to create a scalable logic system from self-assembled gates, we begin to investigate feedback and gain mechanisms as well as optical interconnection at micron scales to make hybrid micro-nano systems in the laboratory. In this section we provide a brief background on DNA self-assembly, RET logic, feedback and non-linear gain media and optical interconnection which motivate us to create the OLE and its SPICE model, and to evaluate more complex logic functions.

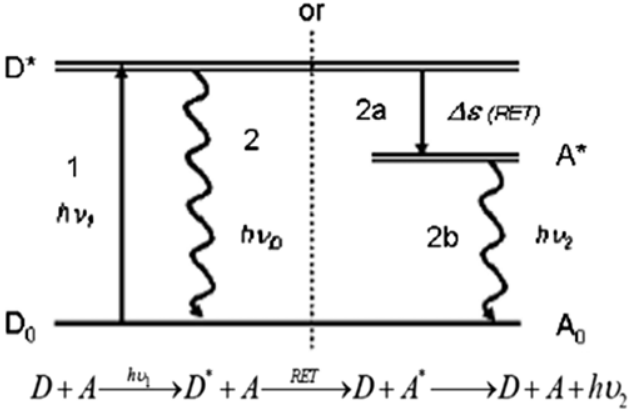

Fig. 2. Transition diagram of RET.

\subsection{DNA self-assembly}

DNA self-assembly is a bottom-up fabrication technique with sub-nm precision that can organize single-molecules into complex and arbitrary patterns. Self-assembly is a spontaneous reaction among strands of DNA that can be controlled by careful sequence design. The entire process is highly scalable and leverages industrial synthetic chemistry which has an infrastructural base the rival of the entire semiconductor industry. In this way, a specific topology of molecules can be created with inter-molecular distances with sub-nm resolution and enormous manufacturing scales (i.e., $>10^{15}$ structures/hour). Fig. 1 illustrates the DNA grid we use as a substrate for RET logic. The motif shown in Fig. 1(a) is composed from nine strands of DNA: one core, four shell, and four arm strands. Grids can be formed by mixing motifs with arm sequences designed to organize finite, square lattices as shown in Fig. 1(b)-(d). Precise chemical patterns, Fig. 1(c), can be made by modifying select motifs, e.g., by modifying one of the nine DNA strands, prior to assembly into a lattice. DNA grids can implement energy transfer circuits that function similar to diode-diode logic by organizing chromophores into geometric patterns. Chromophores are small molecules that absorb light at one wavelength and emit at a different lower energy wavelength [21]. The next section describes the underlying resonance energy transfer phenomena, and how to build RET logic.

\subsection{RET logic}

In this section, we introduce the basic process used in our proposed system to perform computing-Resonance Energy Transfer (RET). First, we describe the details of the RET process and how it can be used to construct logic gates. Next, we demonstrate a RET logic gate we fabricate in a lab environment. Then we compare the area density of RET logic gates with the state-of-the-art CMOS process.

\subsubsection{Resonance energy transfer}

Resonance energy transfer is the foundation for the logic circuits in the proposed system. Fig. 2 illustrates the RET process that occurs between a donor, which is excited either electrically 


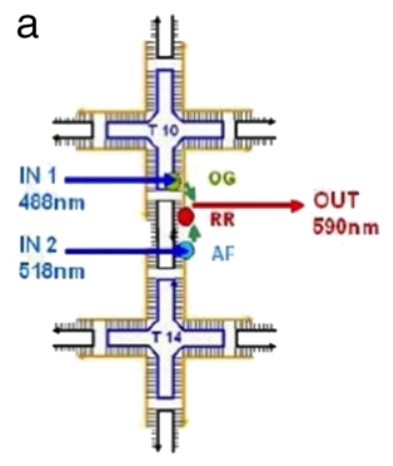

\begin{tabular}{|c|c|c|}
\hline $\begin{array}{c}\text { Input 1 } \\
(488 \mathrm{~nm})\end{array}$ & $\begin{array}{c}\text { Input 2 } \\
(518 \mathrm{~nm})\end{array}$ & $\begin{array}{c}\text { Output } \\
\text { intensity } \\
(@) \text { 590nm })\end{array}$ \\
\hline OFF & OFF & 0 \\
\hline OFF & ON & 31 \\
\hline ON & OFF & 39 \\
\hline ON & ON & 70 \\
\hline
\end{tabular}

Fig. 3. The RET AND-gate. (a) DNA nanostructure layout. (b) Output vs. input.

or optically, and an acceptor which receives energy. Fig. 2 illustrates two possible scenarios for RET in a simple two chromophore (Donor/Acceptor) system. The process begins when the donor $(D)$ absorbs energy, e.g., from a photon, and enters an excited state, $D^{*}$, creating a molecular exciton (step 1 in Fig. 2. Two processes may occur once the exciton is created: (i) a radiative process by which the exciton relaxes to the D0 ground state releasing a photon (step 2), and (ii) a non-radiative process by which the exciton quantum mechanically transfers to the acceptor $(A)$ to create $A^{*}$ (step 2a). The exciton transfer process does not strictly involve energy loss, however, if the acceptor and donor bands overlap but are offset such that the acceptor has a lower energy gap, the exciton will often thermalize (i.e., dissipates energy), over a characteristic period of time, to the lower energy after the transfer event. In this fashion the transfer process is unidirectional from donor to acceptor. Through spontaneous decay $A^{*}$ will relax to $A 0$ by emitting a photon, again over a characteristic period of time, which can then be observed, or "waveguided" to other inputs. The emitted photon usually has a longer wavelength (i.e., lower energy) than the input donor photon because of the relative band structure between $D$ and $A$.

The rate of transfer between the donor and acceptor is largely governed by their separation, relative orientation, and band overlap. By controlling the separation and relative orientation between the chromophores attached to a grid, and by also choosing a pair with sufficient band overlap, it is possible to tune the exciton transfer rate. We can generalize this approach to any number, or even a ratio, of donor and acceptor pairs so long as the donors can be independently excited.

The implementation of RET logic gates requires tuning the exciton transfer rate between multiple donor "inputs" and a single acceptor "output". If the possible rate of transfer is very high between each donor and the acceptor then any single donor input can excite the acceptor output to a prescribed level. This implements an OR-gate because any donor exciton (i.e., logic 1 at an input) will generate an acceptor exciton (i.e., logic 1 at the output). However, if the transfer rates between donors and the acceptor are tuned to be very low then every donor must be excited (i.e., logic 1 on all inputs) to generate sufficient acceptor output (i.e., logic 1 output). This implements an AND-gate because the acceptor output will only be above the prescribed level when each input is excited. Next, we describe preliminary results demonstrating the fabrication of RET gates in the lab environment.

\subsubsection{RET gate demonstration}

This section presents evidence demonstrating the feasibility of using RET to build logic gates. Prior work with integrated chromophores on DNA scaffolds has demonstrated that RET can be used to implement simple logic gates $[27,26]$. We recapitulate the experimental evidence for RET gates here.
Fig. 3 illustrates the design for a RET logic AND-gate. The structure is formed by attaching three chromophores: (1) Oregon Green $^{T M}(\mathrm{OG})$ and (2) Alexa Fluor ${ }^{T M} 535$ (AF 535) as inputs and (3) Rhodamine Red (RR) as an output. The two inputs are excited by photons with wavelengths $488 \mathrm{~nm}$ for OG and $518 \mathrm{~nm}$ for AF 535. Custom spectrofluorometry hardware is required to excite both inputs and observe the output (RR) at an emission wavelength of $590 \mathrm{~nm}$. The measured output at $590 \mathrm{~nm}$ under the four binary input conditions is shown in Fig. 3(b). By applying a threshold to the output, the condition when both inputs are present can be interpreted as logic 1, while the conditions with fewer inputs asserted can be interpreted as logic 0 . This demonstrates that the RET between inputs and output chromophores is sufficiently weak to create logical AND. The OR-gate can be created in a similar fashion, however, the same function can be created more simply by directly observing those inputs with an analog (summing) detector.

\subsubsection{Area density comparison}

To better understand the advantages of the RET logic gates, we compare their area density with state-of-the-art CMOS process. Take the $60 \mathrm{~nm} \times 60 \mathrm{~nm}$ DNA grid shown in Fig. 1(b) as an example. We can allocate 56 RET gates with up to 5 inputs in total. We conservatively assume all the RET gates have only 2 inputs to favor the CMOS process. A 2-input AND gate in CMOS is composed of 4 transistors. Therefore, the area for the logic which equals one transistor in RET-based technology is $16.1 \mathrm{~nm}^{2}$. The area of a 6TSRAM cell is $0.051 \mu \mathrm{m}^{2}$ [30] of the state-of-the-art CMOS process, which means the area of one transistor is $8,500 \mathrm{~nm}^{2}$. Comparing the transistor area between the two technologies, we can see that the density of RET-based technology is at least $100 \times$ better than that of CMOS.

We can use RET to build logic gates such as AND or OR gates with high area density. However, RET is a lossy process as mentioned in Section 2.2.1. Not only is the output wavelength longer (lower energy) than that of the input, but also the output intensity is smaller. It is important to have a mechanism which supports feedback and gain between gates. The next section describes a couple optical devices which are good candidates for this purpose.

\subsection{Feedback and gain media}

To restore the intensity and frequency of RET logic's output signal, feedback and gain media are needed. The feedback media should be able to blue-shift the incident light's longer wavelength back to the desired shorter wavelength range. The light with the shorter wavelength can then be fed into an input of the next level's RET gates. The gain media should have a non-linear intensity response to the incident light to reduce the noise margin. To be more specific, the gain is negligible when the incident light intensity is below a threshold, and the gain is at the required level 

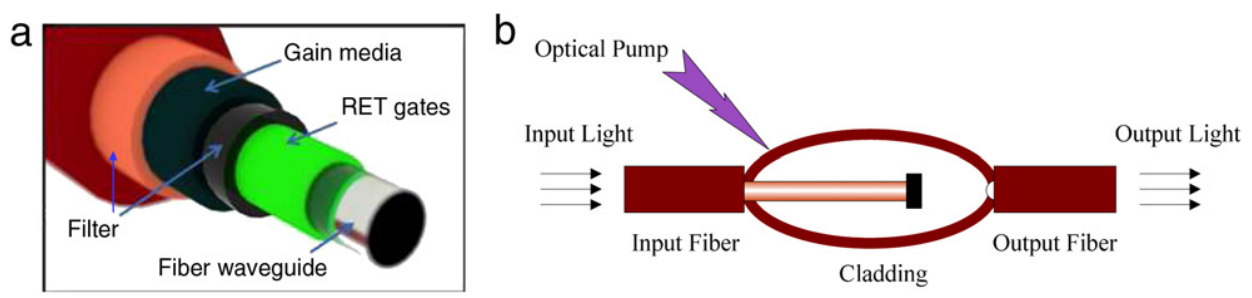

Fig. 4. Optical system overview (a) Layered structure (b) Fiber based optical system.

when the incident light intensity is beyond the threshold. A step function of gain value over light intensity would be the perfect case.

For feedback media, a layer of quantum dots is a potential candidate. Quantum dots have been commonly used in light emitting devices. Because of quantum confinement effects, the emission wavelength can be dramatically tuned by controlling the size of the dots $[1,2]$. One typical way to obtain emission light with a higher energy than the excitation light's energy, which is also called up-conversion, is through a process of two photon absorption (TPA). During the TPA process, two photons of the same or different frequencies simultaneously excite a quantum dot from a lower-energy state to a higher-energy state. The energy difference between the two states is the sum of the two photons. Upconversion in quantum dots has been well demonstrated [33,22]. It is feasible to use this mechanism to provide feedback for RET logic.

Quantum dots can also work as gain media. Klimov et al. $[20,19,18]$ demonstrate optical gain in strong confined quantum dot nanocrystal. They present narrowband stimulated emission with a clear threshold behavior. The emission wavelength is tunable with the quantum dot size. An optical laser is required to pump the quantum dots into a high-energy excited state in order to achieve the optical gain. The entire process is in the optical domain. However, we can also get optical gain through the electrical method by converting photons into electrons. The mechanism is similar to the functionality of an image intensifier [14]. It converts photons into electrons through a photocathode, increases the amount of electrons by microchannel plates (MCP), and converts the electrons back to photons with a phosphor screen. Image intensifiers are well developed nowadays. They are CMOS compatible and their components are available in small sizes $[3,8,11]$.

\subsection{Optical interconnection}

Traditional CMOS computing uses metal wires for interconnection. However, optical computing uses waveguides as wires and insulators. Optical waveguides enable wavelength division multiplexing (WDM) where different wavelengths share the same physical space in the waveguide. This superposition of signals in a waveguide may allow us to reduce the CMOS wiring overhead. Another advantage of a waveguide interconnect is that the delay is always the speed of light regardless of the interconnect length. This is in contrast to electrical interconnects where the delay is proportional to the transmission length. Due to the above properties, optical waveguides are commonly used in on-chip networks $[15,17]$. Moreover, the smaller size of waveguides, such as nanoscale waveguiding [32] have been demonstrated in recent years.

The technologies presented in this section motivate us to integrate different pieces together to create larger optical systems. The basic optical computing unit based on RET logic of such systems is called an Optical Logic Element (OLE). However, it is complex to evaluate such systems without modeling tools. Further, we are also interested in the potential timing and power characteristics of integrated RET logic to understand the possible limitations of the technology. Therefore, we propose a SPICE model that captures the transfer of energy within a RET cascade, interactions between photons and waveguides, and the behavior of feedback and non-linear gain media. The SPICE model enables us to evaluate more general systems [34,5], and its outputs can serve as a set of technology parameters for a larger-scale computer architectural system simulation framework. In the next two sections, we present the OLE design and its SPICE model in detail.

\section{The optical logic element}

Inspired by the RET logic and other technologies in Section 2, we design a basic computing unit called the Optical Logic Element (OLE) which uses a layer of RET logic for computation. In this section, we present the structure of OLE systems, preliminary results of integrating RET logic on a fiber setup, and circuit design with OLEs.

\subsection{Structure of optical logic element system}

An OLE is functionally equivalent to a Lookup Table (LUT) with up to 5 inputs implemented by a collection of AND-gates which create an arbitrary set of minterms. In principle, arbitrary numbers of input and output chromophores could be chosen for RET gates. However, in practice, this number is constrained by the compatibility of available chromophores, the DNA grid properties, such as limited locations for attaching chromophores, the feasibility of donor-acceptor pairs and pairwise transfer rates. We have demonstrated logical AND/OR gates as shown in Section 2.2.2, but they do not create a complete logic set without a NOT gate. Ongoing work is focused on developing the NOT gate. Since we have not yet implemented an inverter, OLEs use dual-rail encoding of signals to form a logically complete set of functions. Dual-rail logic encodes a single Boolean input value with two independent signals that are complementary, in the case of RET logic this means two distinct wavelengths of light for each input. Dual-rail outputs must be generated from the true and complement of the original single-rail function, thus consuming twice the hardware. This overhead is acceptable since the density of RET logic is substantially higher than optical waveguides or fibers.

An OLE is a layer-by-layer structure. With a fiber optic as an interconnection method as shown in Fig. 4(a), the four layers from the bottom to the top are defined as:

- RET-logic gate layer: implements all minterms with up to five inputs. This layer is responsible for computing.

- Input color filter layer: absorbs input photons to prevent them from stimulating the feedback and gain media layer. A color filter implements an optical low or high pass band made from organic dyes, such as chromophores.

- feedback and non-linear gain media: provides signal restoration and optical gain. Potential candidates for this layer include quantum dots, micro Photo Multiplier Tubes ( $\mu$ PMT) which include photocathodes and Microchannel Plates (MCP), or any other small size devices which restore the signal's frequency and intensity. If we use a quantum dot layer as an example, this layer absorbs light from optical pumps to provide the necessary energy for up-conversion (i.e., long to short wavelength 


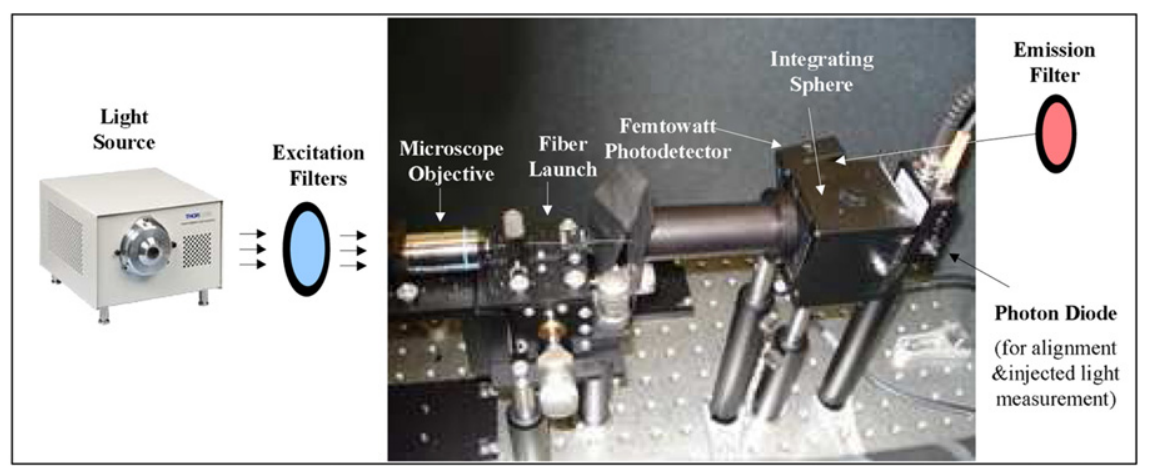

Fig. 5. The RET AND-gate. (a) DNA nanostructure layout. (b) Output vs. input.

conversion) and producing multiple output photons from each incident minterm photon, i.e., gain.

- Output color filter: removes un-absorbed output photons from the minterms and passes the intensified minterm outputs from the feedback and gain media layer.

We propose a complete optical system composed of OLEs with a fiber optic realization as shown in Fig. 4(b). The system is composed of several parts: an input and output filter, a cladding wrap, an input fiber core coated with a layer of OLEs to implement logic functionality, optical pumps, and input and output light sources.

Signals in terms of photons with different wavelengths are injected into and collected from fibers which could be directly connected to a communications network. The fibers serve as wires and insulators and provide connectivity between OLE outputs and inputs. Signals can merge onto one fiber by applying "join" techniques that bundle multiple fibers into one, similar to on-chip optical waveguide techniques [10]. Similar techniques exist for splitting, i.e. the fan-out of, signals. Fig. 4(b) illustrates a wrapper of cladding that works similar to an integrating sphere and collects emitted light from OLE outputs to an output fiber. Optical pumps are used for restoration of optical signals' frequency and intensity. For large-scale fabrication and on-chip applications, the fibers can be replaced by waveguides which serve the same function.

Next, we present some preliminary results of integrating RET logic into fiber optics.

\subsection{Preliminary results of OLE demonstration}

Prior work with integrated chromophores on DNA scaffolds shows that RET can be used to implement simple logic gates $[27,26]$ (Section 2.2.2). Furthermore, most components of OLEs including quantum dots [1,2,33,22], waveguides [32] and color filters [21] have been individually demonstrated and are all silicon compatible. The only component that has not been demonstrated is the evanescent coupling of chromophores to waveguides. Toward a complete integrated technology of RET logic, in this section we show early results of integrating RET circuits on fiber optic cables as a convenient, and generalized, stand-in for microscale, on-chip optical networks [15,23,31]. We develop a prototype fiber-based system which can characterize the behavior of RET logic gates integrated into a fiber optic cable. The fiber serves as both the substrate and input waveguide for the gate.

Optical inputs with various wavelengths can be inserted into the same waveguide by superposition (i.e., photons can occupy the same space) and each can selectively excite the RET gate if it is within 10-50 $\mathrm{nm}$ of the fiber optic core by evanescent wave coupling. To prepare the fiber we first strip the cladding and buffer coat of a commercially available $400 \mu \mathrm{m}$ core quartz fiber, and then use dip- or drop-coating of the RET gates to apply one or more layers of AND-gates to the exposed core. To improve the coupling efficiency we also employ oxygen plasma to clean the stripped fiber ends. Upon injection of input photons the RET gates emit output photons radiating outward from the sidewall of the fiber core according to the transfer function shown in Fig. 3(b).

The experimental setup to inject inputs and observe output is shown in Fig. 5. An incandescent broadband, i.e., white, light source passes light through Bragg interference filters to select the input wavelengths under test. The filtered light, a combination of any relevant input wavelengths, is injected into the fiber through a microscope objective aligned to focus the light onto the backend of the fiber (i.e., the side without RET gates). The active end of the fiber (with RET gates) is inserted into an integrating sphere that captures any emitted photons and focuses them on a femtowatt-sensitive detector and emission filter (to select an output wavelength and measure the fluorescence intensity).

We demonstrate several proof-of-principle experiments on fibers with single chromophores, single chromophores attached to $2 \times 2$ DNA grids, and a donor/acceptor pair. Fig. 6(a) shows a Gaussian fit to the fluorescence intensity of Oregon Green ${ }^{T M} 488$ excited by different wavelengths of light observed through a $532 \mathrm{~nm}$ emission filter. This curve matches the expected excitation spectrum of Oregon Green ${ }^{T M}$ provided by the vendor as shown in Fig. 6(b). This demonstrates the coupling of chromophores to a waveguide and the system's capability of reliably detecting chromophores.

We also test the system for its ability to detect RET between donor-acceptor pairs dip-coated onto a fiber. To do this we use Oregon Green ${ }^{T M} 488$ as a donor and Alexa Fluor ${ }^{T M} 594$ as an acceptor and coated a freshly cleaned fiber. Fig. 7 shows the fluorescence intensity of two fibers with a donor-acceptor pair prepared as described above. Upon injection of two intense inputs (543 and $441 \mathrm{~nm}$ ) the pairs exhibit time-varying wavelength dependent behavior. Photons at $543 \mathrm{~nm}$ will only excite the acceptor and photons at $441 \mathrm{~nm}$ will excite both the donor and acceptor and the comparison of the relative decay of each as a function of excitation wavelength can confirm RET.

After a period of about 20 min of excitation at $543 \mathrm{~nm}$, the donor's emission fluorescence intensity increases and the acceptor's decreases due to photobleaching. However, this is not the case when the pair is excited by $441 \mathrm{~nm}$ light. The wavelength dependent response of the pair can be explained by considering that RET transfers energy from an excited donor to an acceptor and this prevents the donor from emitting a photon. If we reduce the effect of RET by bleaching the acceptor, i.e., by using intense inputs over long periods of time, we can observe greater emission from the donor. Further, if we bleach both the donor and acceptor, both their emissions are reduced. Another set of experiments further proves the detection of RET by showing that the bleaching rate of the donor without the accepter is faster than with the acceptor (data not shown) as expected.

The preliminary experimental results presented here show that fiber optic systems are a practical and convenient framework for 

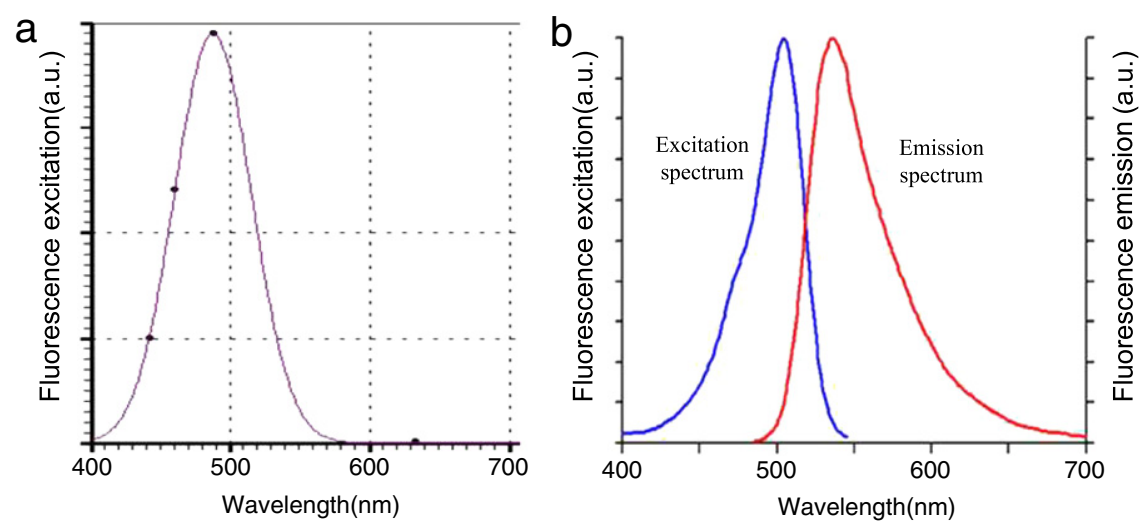

Fig. 6. Excitation spectrum of Oregon Green ${ }^{T M} 488$ Chromophore from (a) Gaussian fit to the measured fluorescence Intensity, and (b) vendor.

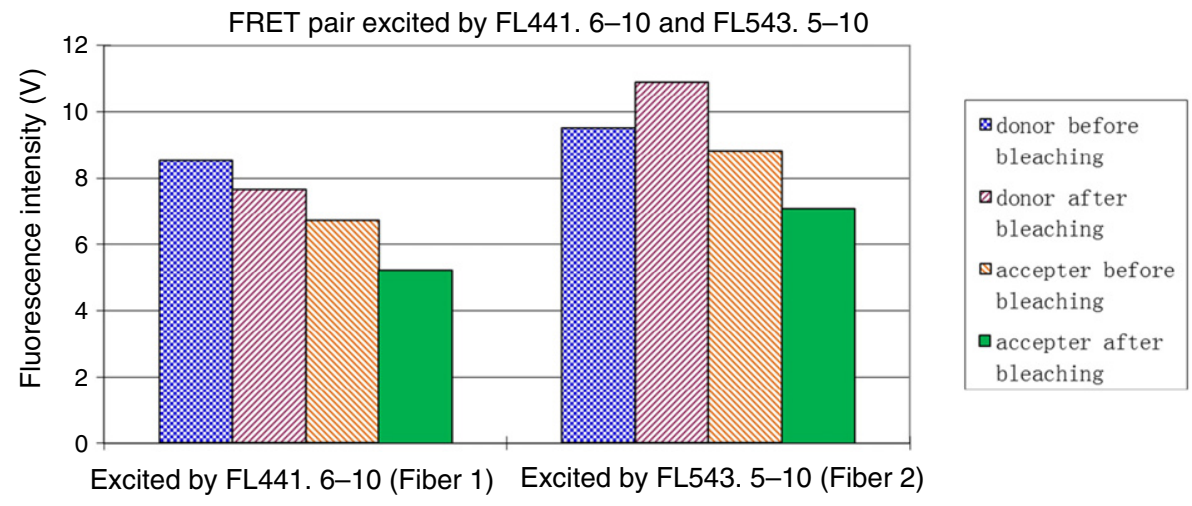

Fig. 7. FRET pair emission fluorescence intensity comparison excited by different wavelengths.

the study of RET logic and the integration of such into larger systems. It is feasible to build an OLE-based optical system in such a fiber setup. Other integration and experimental challenges include integrating the feedback and gain media into the prototype system and 3D fabrication of the system [31,4].

\subsection{Circuit design with OLES}

We follow a few rules when designing circuits with OLEs. First, each minterm/OLE has up to five inputs; Second, each circuit requires both true and complementary logic design, because OLEs use dual-rail encoding; Third, the entire system has up to ten different wavelengths to choose from for the dual-rail encoding in order to avoid crosstalk between different OLEs. To design any circuit with OLE, we can simply take three steps: (1) logic expression optimization, (2) minterm and OLE mapping, and (3) wavelength mapping. In this section, we give a couple examples of using OLEs to design both combination and sequential circuits.

\subsubsection{2-Input multiplexer design}

To design a 2-input multiplexer with input $A$ and $B$, first we get the logic expressions for both the true and complementary output $O$ and $\bar{O}$ as shown in the left part of Eqs. (1) and (2):

$O=S A+\bar{S} B=>O L E_{T}=$ minterm $1+$ minterm 2

$\bar{O}=\bar{S} \bar{B}+S \bar{A}+\bar{A} \bar{B}=>O L E_{C}=$ minterm 3

$$
+ \text { minterm } 4+\text { minterm } 5 \text {. }
$$

To better map them to OLEs, Sum-of-Products (SOP) expressions are preferred. Second, map the sum to an OLE and each

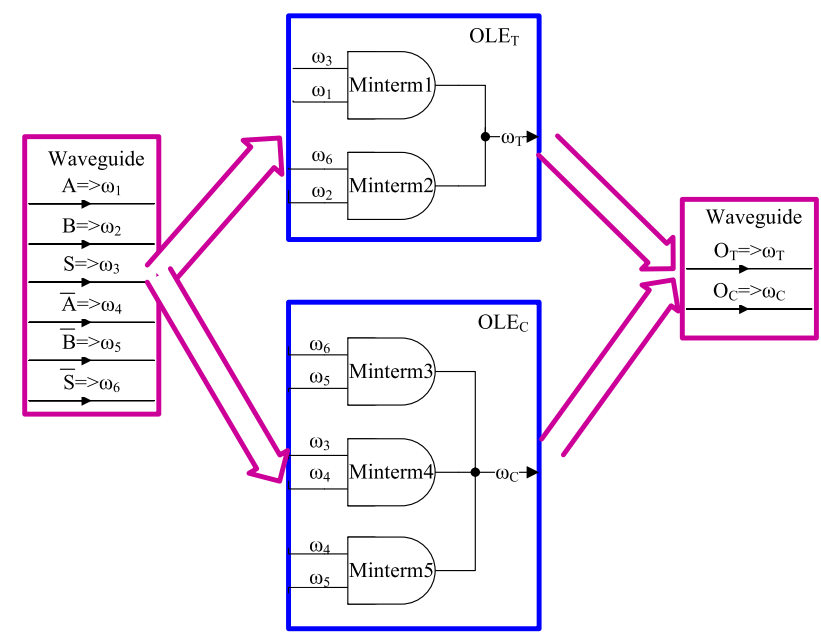

Fig. 8. 2-input multiplexer design with OLEs.

distinguished product to a different minterm. Since one OLE functionally equals a LUT with up to five inputs, one OLE will be enough to compute the true or the complementary output in this example. The mapping results are shown in the right part of the Eqs. (1) and (2). OLE $E_{T}$ is the output of the true logic of the multiplexer and $O L E_{C}$ is the complementary circuit. In the end, we map all the inputs and outputs to different optical wavelengths as shown in Fig. 8. The input waveguide splits the signals into true and complementary OLE and after calculation, the output true signal $\omega_{T}$ and complementary signal $\omega_{C}$ join back to the output waveguide. 
a
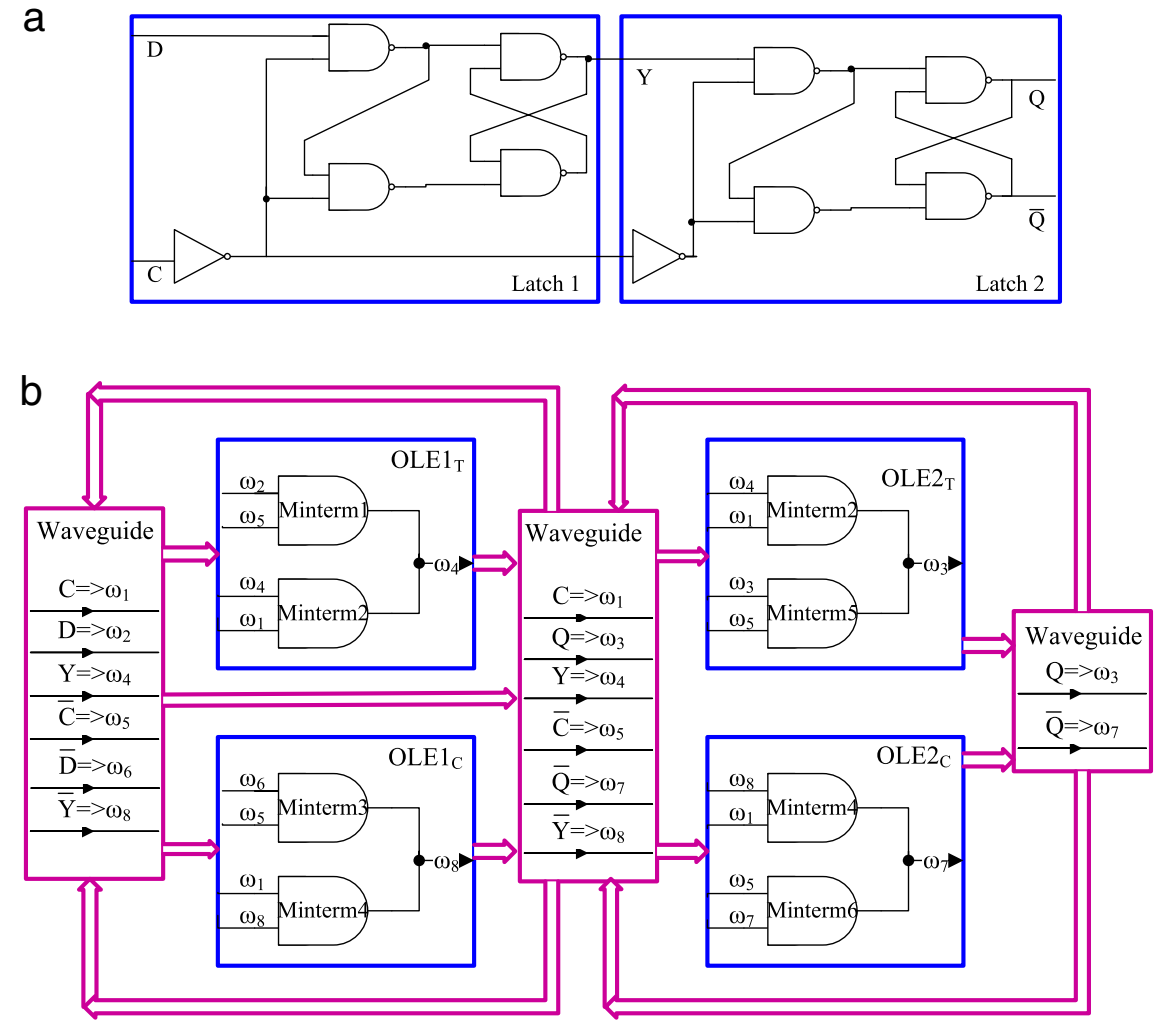

Fig. 9. DFF circuit design with (a) CMOS logic gates, and (b) OLEs.

The circuit of 2-input multiplexer is relatively simple. It does not have feedback, so we do not need to map the output wavelengths $\omega_{T}$ or $\omega_{C}$ back to any input wavelength. The number of inputs is less than five, so the computation can be handled by one level of OLEs. However, for circuits with more than five inputs, we need to break down the logic into multiple levels of OLEs.

\subsection{2. $D$ flip-flop design}

The previous section shows an example of combinational circuit (2-input multiplexer) design with OLEs. In this section, we present an example of sequential logic design-a master-slave positiveedge-triggered D flip-flop (DFF). The circuit of DFF is shown in Fig. 9(a). A DFF has two gated D latches in series and each latch has a truth table as shown in Table 1. To implement DFF with OLEs, first, we obtain the logic expressions for two levels of D latches in the left parts of Eqs. (3)-(6). Latch 1 is

$Y=D \bar{C}+Y C=>O L E 1_{T}=$ minterm $1+$ minterm 2

$\bar{Y}=\bar{D} \bar{C}+\bar{Y} C=>O L E 1_{C}=$ minterm $3+$ minterm 4

and Latch 2 is

$Q=Y C+Q \bar{C}=>O L E 2_{T}=$ minterm $2+$ minterm 5

$\bar{Q}=\bar{Y} C+\bar{Q} \bar{C}=>O L E 2_{C}=$ minterm $4+$ minterm 6 .

Then we map each SOP of the two latches to an OLE as shown in the right part of Eqs. (3)-(6). Each distinguished product is also mapped to a different minterm. Since $Y C$ and $\bar{Y} C$ appear twice, minterm 2 and minterm 4 are reused. In the end, we map inputs and outputs to proper wavelengths as shown in Fig. 9(b). Since a DFF has feedback signals, each OLE's output signal needs to be mapped back to the corresponding input wavelength, such as $\omega_{3}, \omega_{4}, \omega_{7}$, and $\omega_{8}$.
Table 1

Truth table of a gated D latch.

\begin{tabular}{llll}
\hline $\mathrm{D}$ & $\mathrm{C}$ & $Y$ & $\bar{Y}$ \\
\hline 0 & 0 & 0 & 1 \\
1 & 1 & $Y$ & $\bar{Y}$ \\
0 & 1 & $Y$ & $\bar{Y}$ \\
1 & 0 & 1 & 0 \\
\hline
\end{tabular}

The two above examples show how to design combinational and sequential circuits with OLEs. Although the two circuits presented here are simple, any bigger circuit can be broken down into such simple circuits, and then follow the three steps to obtain the optimized logic expression, map OLEs and minterms, and then map wavelengths. Therefore, the OLE system is capable of performing general computations and implementing any functionality. It can complement or replace functionality currently implemented in silicon. In the next section, we present the SPICE model for the OLE system in detail.

\section{The SPICE model for optical logic elements}

To evaluate the OLE systems including timing and power characteristics and to better understand the possible limitations of the technology, we build a SPICE model for OLEs. In this section, we first introduce the SPICE model of all the components including the fiber, minterms, color filters, feedback and gain media space (quantum dots in the model), and some features such as decay and Gaussian noise. We then present the validation of the SPICE model.

\subsection{SPICE model of the OLE and its components}

The SPICE model we create for the entire OLE optical system is shown in Fig. 10. The detailed structure of minterms and OLEs are 


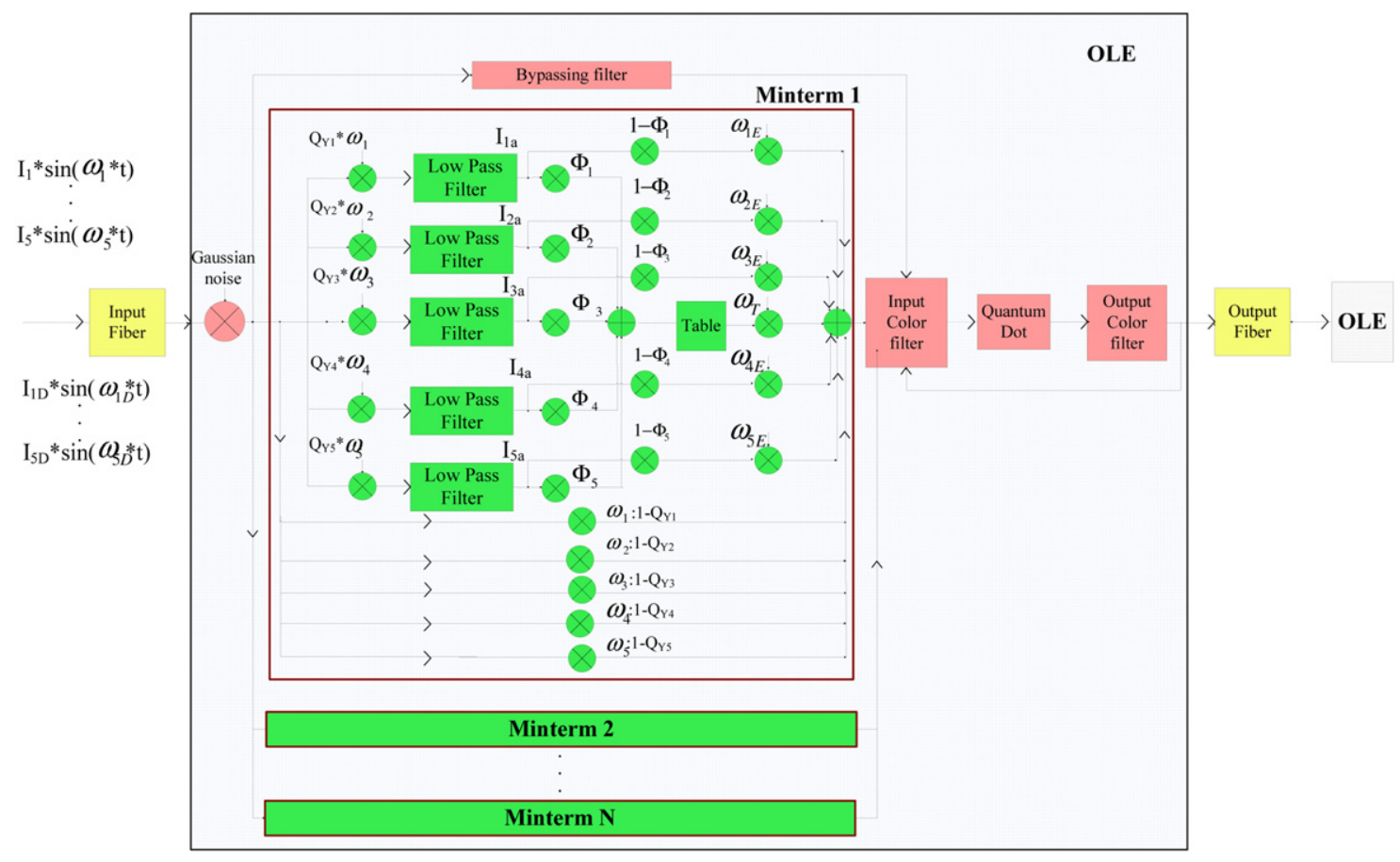

Fig. 10. SPICE model structure of the optical system.

shown in the two large squares. The SPICE model uses scaled time and sinusoidal sources to represent photons propagating within a waveguide. Beyond the models the larger system requires each OLE to bypass optical signals which are not absorbed by any intentional filter and thus should physically bypass the element. In principle this part of the model tests our choices for the input and output color filters and couples non-ideal noise sources across the OLE.

The SPICE model of an OLE is flexible. We can either use it as a complete unit to evaluate circuits composed of OLEs, or we can pick a few pieces of interest and use it as a customized model. For instance, we can build lossy circuits with minterms followed by color filters without the feedback and gain media layer. Moreover, the model is fully parameterized. Various chromophores are modeled by changing their quantum efficiency, lifetime, etc. A different non-linear gain media such as $\mu$ PMT, as mentioned previously, can replace the quantum dot layer by changing the gain intensity, propagation delay, etc. Without loss of generality, in the rest of the paper, we use quantum dots as the feedback and gain media in modeling and simulation. It is worth noting that the model also captures the timing information such as propagation delay, rise and fall time etc. of both sequential and combination circuits as shown later in Section 5. However, due to lack of information in literature, the timing of gain media might not be modeled precisely. Gaussian noise in spectrum for both excitation and emission light is also modeled. Next, we present the SPICE model for each component and some important features such as decay and Gaussian noise.

\subsubsection{Waveguide}

A band pass filter can emulate the fiber's or waveguide's transfer function. The input of the filter takes the merged signals as input to the OLE and is implemented as an ideal band pass filter in the SPICE model. We assume waveguide attenuation is achromatic thus simplifying the SPICE model.

\subsubsection{Minterms}

Minterms are implemented by collections of a few thousand RET logic AND-gates selected to respond for each minterm input combination. To model this in SPICE we reduce the RET process to modulation and filtering of time-scaled sinusoidal signals which represent photons in the system. First, the combined input signal (i.e., all incident photons) is demodulated with a carrier that represents the excitation spectrum of the donors. Following this, a lowpass filter extracts a DC component which indicates the intensity of that donor's excitation signal. Finally, the DC component is used to amplitude modulate the acceptor's emission spectrum to produce an output. This model correctly captures the steady-state behavior of collections of donor-acceptor pairs. A preset threshold is applied to the lowpass filter output to model the tuning of RET between input donors and acceptors. When the $\mathrm{DC}$ component rises above this threshold the acceptor signal is maximal indicating logic 1 , otherwise it is too low for an appreciable signal to pass the input filters indicating logic 0 . We use a table function from a Semi-empirical SPICE simulator [9] to set the target intensity.

Each minterm has three parameters for RET, shown in Eqs. (7)-(9). The first is quantum_efficiency, which is the ratio of emitted photons to the total number of absorbed photons. The other two parameters, RET_emission_ratio and direct_emission_ ratio, correspond to the ratio of total absorbed photons participating in non-radiative and radiative processes, respectively. Photons un-absorbed by the minterms are directly emitted and blocked by the input color filter. All three parameters are implemented by voltage-controlled voltage sources in SPICE.

$$
\begin{aligned}
& Q_{y}=q u a n t u m \_e f f i c i e n c y=\frac{\# p h o t o n \_e m i t t e d}{\text { \#photon_absorbed }} \\
& 1-\Phi=R E T \_e m i s s i o n \_r a t i o=\frac{\text { \#photon_RET_emission }}{\text { \#photon_total_emission }} \\
& \Phi=\text { direct_emission_ratio }=\frac{\text { \#photon_direct_emission }}{\text { \#photon_total_emission }} .
\end{aligned}
$$

\subsubsection{Color filters}

Two layers of color filters eliminate unwanted signals within the OLE. The first layer nearest the minterms, or the input color filter, is a bandpass filter centered on the effective wavelength 


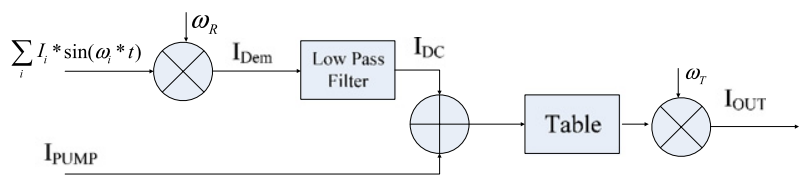

Fig. 11. Structure of quantum dot SPICE model.

of the minterm output. The second layer, or output color filter, eliminates every internal signal except the up-converted output from the quantum dot layer. Through the use of color filters the OLE will only be sensitive to input wavelengths and emit a single output wavelength.

\subsubsection{Quantum dots}

A quantum dot (QD) layer $[1,2,20]$ is used to increase the intensity of and up-convert the minterm signal. These processes require energy which is supplied by external optical pumps. The SPICE model for this layer is shown in Fig. 11. The basic structure is similar to the minterm model, i.e., demodulation-lowpass filter-modulation. To model the gain, a DC pump value is added to the lowpass DC output to bring the sum above a threshold. The table function and modulation part have the same function as in minterms and are used to set the output intensity and wavelength of the output.

The time-dependent behavior of the quantum dot layer is modeled similarly. In this case we use a first order RC circuit to implement the delay and by setting a threshold smaller than the full voltage magnitude on the capacitor, we are able to achieve longer discharging times than charging times.

\subsubsection{Decay}

To better characterize the time-dependent features of RET we also model the excited lifetime of chromophores and quantum dots as charging and discharging in the SPICE model. Charging represents the onset of fluorescence due to the absorption of light or RET, upon which the fluorescence of the acceptor grows exponentially. Discharging represents the heavy-tail exponential decay of excitons at the acceptor which leads to fluorescence decaying exponentially in time. The discharging time is usually longer than the charging time due to the longer lifetime of the acceptor. These times are the fundamental performance limiters of the OLE and are typically pico to nanoseconds. The lifetime of some chromophores can be as low as 0.2 ps [24].

\subsubsection{Gaussian noise}

Due to line splitting, the excitation and emission spectra of chromophores are more similar to Gaussian functions than delta functions in the frequency domain. We include this non-ideality in the SPICE model by considering the Gaussian function and its Fourier transform functions as follows:

$g(t)=\frac{1}{\sigma \sqrt{2 \pi}} e^{\frac{-r^{2}}{2 \sigma^{2}}}$

$g(\omega)=\frac{-\omega^{2} \sigma^{2}}{2}$.

Euler's formula gives us Eq. (12) and its Fourier transform Eq. (13).

$$
\begin{aligned}
& \sin \omega_{s} t=\frac{i}{2}\left(e^{-i \omega_{s} t}-e^{i \omega_{s} t}\right) \\
& F\left(\sin \omega_{s} t\right)=\frac{i}{2}\left(\delta\left(\omega+\omega_{s}\right)-\delta\left(\omega-\omega_{s}\right)\right) \\
& g(t) \times \sin \omega_{s} t=\frac{i}{2} g(t)\left(e^{-i \omega_{s} t}-e^{i \omega_{s} t}\right) \\
& F\left(g(t) \times \sin \omega_{s} t\right)=\frac{i}{2}\left(G\left(\omega+\omega_{s}\right)-G\left(\omega-\omega_{s}\right)\right) .
\end{aligned}
$$

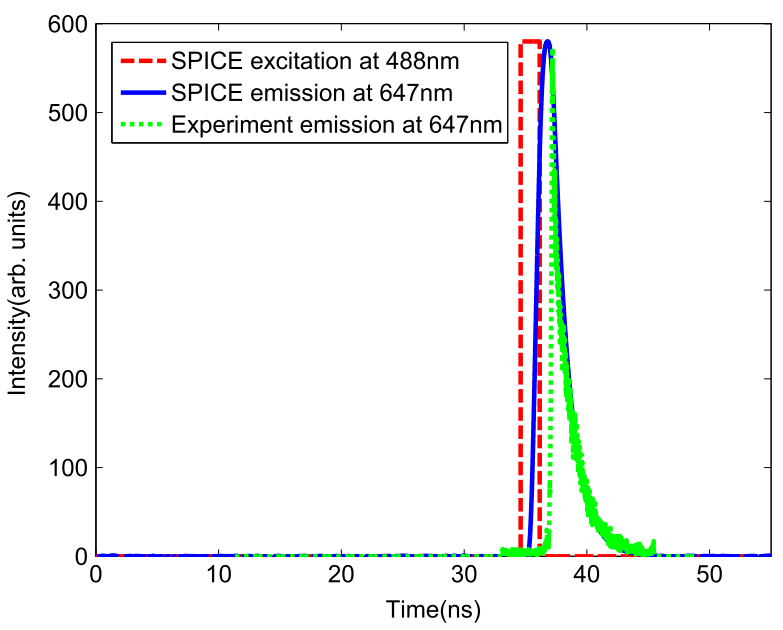

Fig. 12. Experimental and SPICE simulated light emission of a FRET pair AF488 and AF647.

Therefore, as shown in Eqs. (14) and (15), a sinusoidal function multiplied by a Gaussian function in time domain gives us the pulses from the sinusoidal function in the frequency domain multiplied by a Gaussian function. We use a piecewise linear voltage in the SPICE model to represent simulation time and a nonlinear dependent source to calculate Eq. (10) as a node voltage. If we assume donor and acceptor emission bands are comparable in width, we can then set the cutoff frequency of the lowpass filter in the minterms to be larger than half of the Full Width at Half Maximum (FWHM) of the peak in the Gaussian function for this property to propagate to the output signals. Thus, the spectra of the signals in the SPICE model are accurate representations of a real RET system.

\subsection{Validation of the SPICE model}

In this section, we present the validation of the SPICE model for the RET logic-based fiber framework.

The basic computing unit of the SPICE model is an OLE which is mainly composed of minterms, quantum dots and waveguides. To verify the SPICE model, we can primarily verify the correctness of each individual component. Therefore, we create SPICE models for each individual piece and compare them with the experimental results.

\subsubsection{Minterm validation}

To validate the minterm model, we need to verify both the timing and intensity simulation results are correct. To verify the timing behavior, we compare both the experimental and SPICE emission of a Fluorescence Resonant Energy Transfer pair (FRET)Alexa Fluor $^{T M} 488$ (AF488) and Alexa Fluor ${ }^{T M} 647$ (AF647) excited by a short laser pulse at $488 \mathrm{~nm}$ wavelength. First, in a lab environment, we take a cuvette of $90 \mathrm{nM}$ solution of AF488 and AF647 fabricated on DNA grids with a distance of $1.72 \mathrm{~nm}$ between each pair and excite it by a delta pulse from a class IV supercontinuum white light laser with a $488 \mathrm{~nm}$ laser line filter. We calculate quantum_efficiency as $9.60 \times 10^{-6} \%$ according to Beer-Lambert's law [29] and RET_emission_ratio as $99.89 \%$ according to the RET theory [29]. The sample is excited near 37 ns and the emission light intensity follows the dotted line as shown in Fig. 12.

Then we create a SPICE model for the FRET pair with the same parameters-quantum_efficiency and RET_emission_ratio. We excite the pair with a $1.5 \mathrm{~nm}$ width pulse and the emission light intensity of the acceptor follows the solid line as shown in Fig. 12. The SPICE simulation results show a very short 0.5 ns delay 


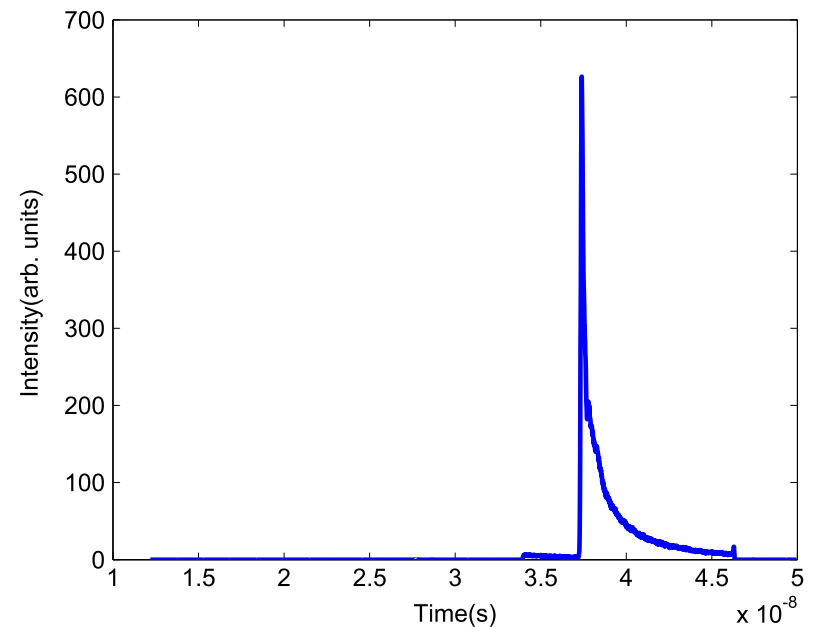

Fig. 13. Experimental emission light of a FRET pair: AF594 and AF647.

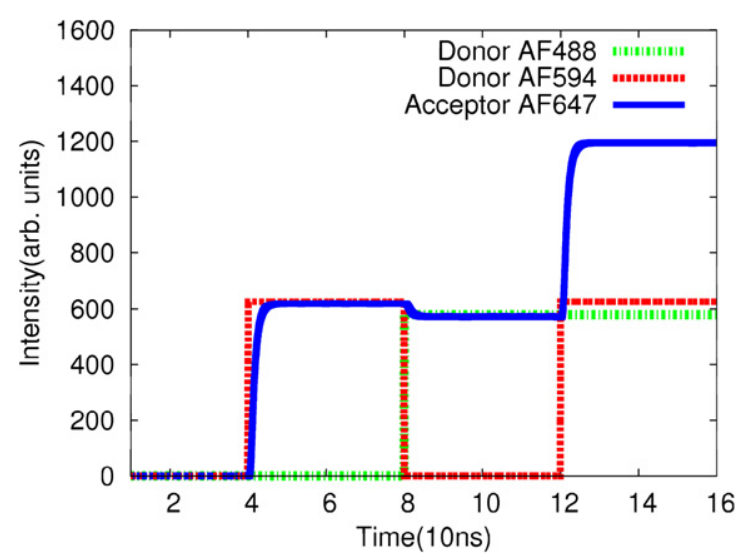

Fig. 14. SPICE simulation of a 2-input minterm M3.

between the excitation and emission and the acceptor's decay follows an exponential curve with a lifetime around $1.3 \mathrm{~ns}$. If we compare the two emission light curves from the experiment and SPICE model, the delay time between emission and excitation, fluorescence onset and decay time are all similar. The only difference is that the SPICE emission curve is a little bit wider than the experimental one. This is because we use a wider excitation pulse in the SPICE model to allow the decay circuit, which utilizes RC delay, to fully charge the capacitance.

To validate the intensity of the minterms, we apply parameters from lab experiments of two FRET pairs with the same acceptor to a 2-input minterm M3, which is a 2-input AND gate, in the SPICE model. Then we run the simulation of M3 when the inputs vary to see if the simulation results match the experiments or not. We first measure the emission light of another FRET pair of Alexa Fluor ${ }^{T M}$ (AF594) and AF647 with the same experimental setup as the AF488 and AF647 pair, except that the excitation light is a laser pulse with wavelength $543 \mathrm{~nm}$. The experimental result is shown in Fig. 13. The quantum_efficiency is $1.39 \times 10^{-5} \%$ according to Beer-Lambert's law [29] and RET_emission_ratio is 99.99\% according to RET theory [29].

We then build a SPICE model for the M3 minterm composed of two donors - AF488 and AF594 and one acceptor - AF647. We apply the experimental parameters such as quantum_efficiency etc. to the SPICE model and get the simulation results as shown in Fig. 14. Fig. 14 shows the acceptor's emission intensity when the inputs intensities varies from logic '00', '10', '01' and ' 11 '. When only

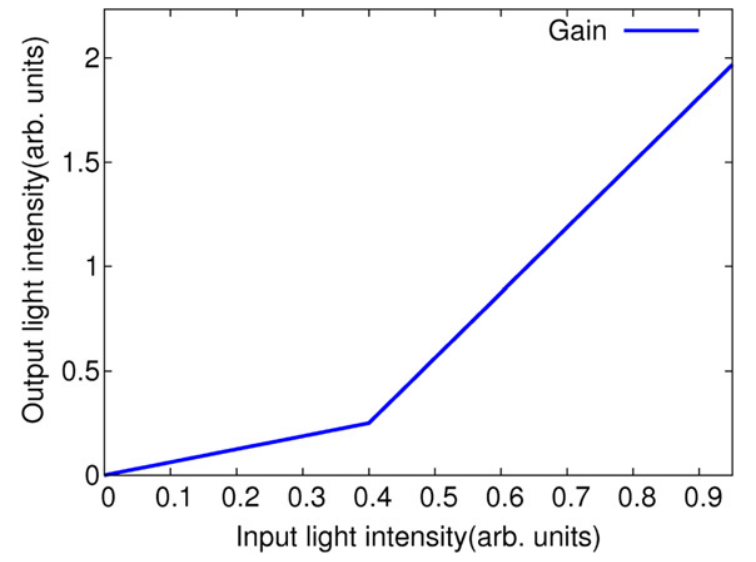

Fig. 15. The non-linear transfer function of the quantum dot gain media.

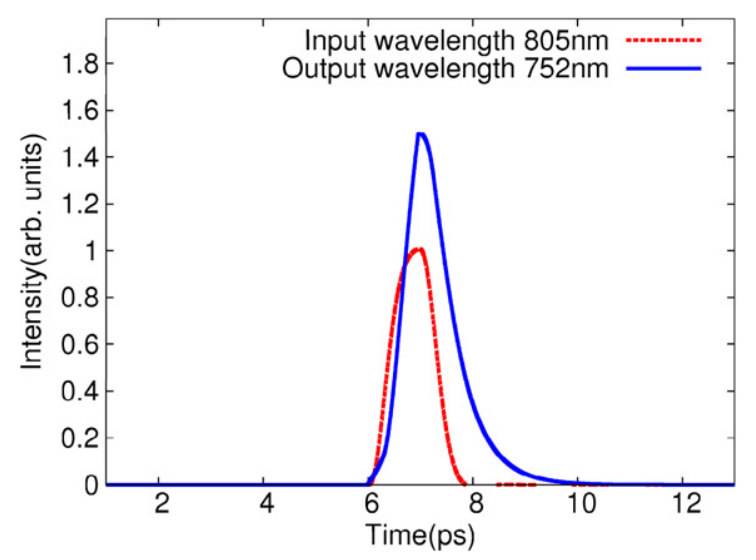

Fig. 16. Stimulated emission of the quantum dot layer.

one input has logic ' 1 ', the acceptor's emission is similar to a single FRET pair's emission from experiments. When both the inputs have logic ' 1 ', the emission intensity of the acceptor is the sum of the acceptor in each individual pair, which is consistent with results presented by Pistol et al. [26]. For an OLE which requires multiple minterms, the output is an OR function of all the minterms outputs. It can be easily implemented by using an analog (summing) detector and thus the results are not presented here.

\subsubsection{Quantum dot validation}

We model a layer of quantum dots in SPICE to provide both feedback and gain media. To validate the correctness of the model, we feed a $1 \mathrm{ps} 805 \mathrm{~nm}$ pulse with intensity of 1 (arb. units) and light from a laser pump with 0.2 intensity (arb. units) and similar width and wavelength into the input of the quantum dot SPICE model. Fig. 15 shows the transfer function of the gain and Fig. 16 shows the stimulated emission light from the quantum dots. If we assume the quantum efficiency of the quantum dot layer is $60 \%$, then the sum of input pulse and the pump light intensity is 0.8 , which is bigger than the threshold 0.4. From the gain media's transfer function. we can get $1.5 \times$ intensity gain, which greatly increases the input light intensity from quantum dots. We also plot the frequency spectrum of signals from Fig. 16 over 13 ps as shown in Fig. 17. We can clearly see that the output wavelength is blue-shifted from $373 \mathrm{THz}$ $(805 \mathrm{~nm})$ to $399 \mathrm{THz}(752 \mathrm{~nm})$. The simulated results are consistent with the experimental results $[33,20,19,18]$.

When we model different circuits in SPICE, we assume that we can customize the quantum dots' transfer function by changing the density of the packed quantum dots $[20,19]$ so that the intensity of 


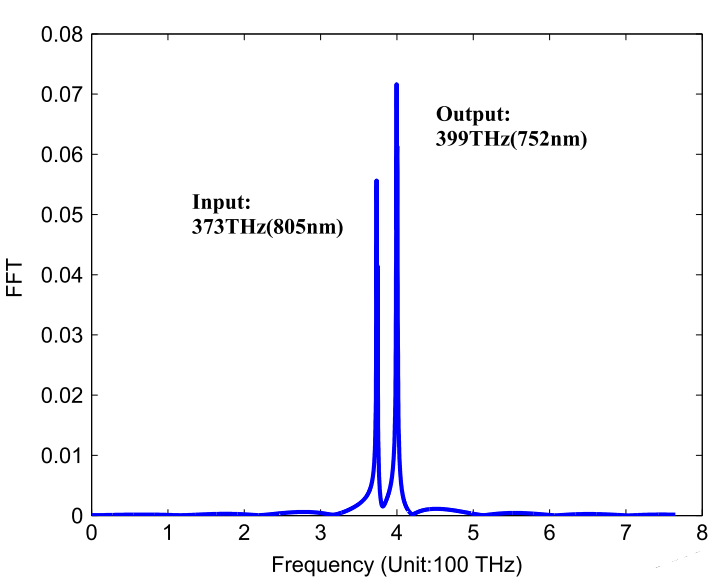

Fig. 17. Frequency spectrum of the input and output of quantum dot layer.

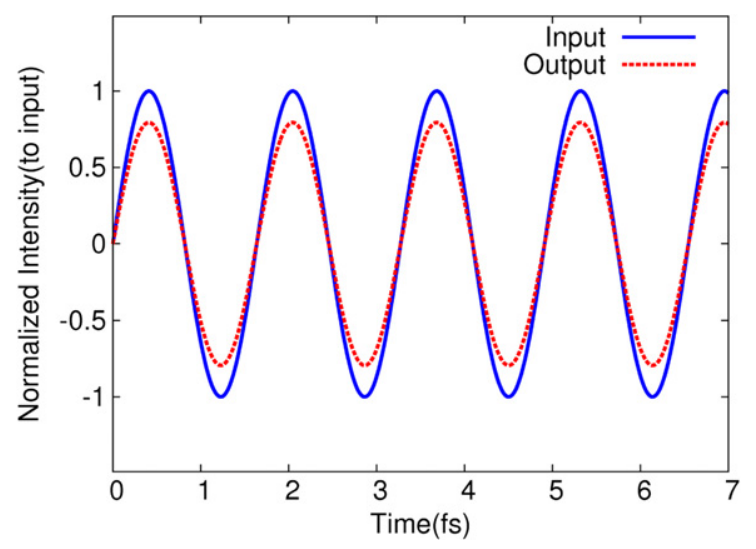

Fig. 18. SPICE simulation results of a $1 \mathrm{~cm}$ long waveguide with $1 \mathrm{~dB} / \mathrm{cm}$ transmission loss.

the OLE output is normalized. This assumption makes the circuit design with OLE cascades much easier.

\subsubsection{Waveguide validation}

To validate waveguide's SPICE model, we take a $1 \mathrm{~cm}$ long waveguide with $1 \mathrm{~dB} / \mathrm{cm}$ transmission loss and model it in SPICE. When input light at $493 \mathrm{~nm}$ wavelength passes through the waveguide, the input and output waveforms are shown in Fig. 18. The output keeps the same frequency of the input but with about 0.8 of input's intensity, which clearly shows the light attenuation by the waveguide.

In conclusion, OLE-based logic circuits can be modeled in SPICE and our validation indicates that the SPICE model results are consistent with experimental results. This means our SPICE model can reproduce the experiment results and our modeling method is valid. In the next section, we show SPICE simulation results of a set of common circuits built with OLEs.

\section{SPICE analysis of OLE circuits}

We design various OLE-based logic circuits including basic combinational and sequential circuits as shown in Table 2. An important circuit in the table is the oscillator which can be used to implement the clocking in sequential circuits. Since we use dualrail encoding, both true and complementary logic for each circuit were designed. The 2 nd and 3rd columns in Table 2 show the total number of minterms and OLEs. In the remainder of this paper, we present both timing and power analysis for the benchmark circuits.
Table 2

Simulation results of benchmark circuits.

\begin{tabular}{|c|c|c|c|c|c|c|}
\hline Benchmark circuit & \#minterms & \#OLEs & $\begin{array}{l}\tau_{p} \\
(\mathrm{ps})\end{array}$ & $\begin{array}{l}\tau_{R} \\
\text { (ps) }\end{array}$ & $\begin{array}{l}\tau_{F} \\
\text { (ps) }\end{array}$ & $\begin{array}{l}\text { Energy } \\
\left(10^{-18} \mathrm{~J}\right)\end{array}$ \\
\hline 2 bit multiplexer & 5 & 2 & 2.4 & 0.63 & 1.2 & 3.11 \\
\hline 1 bit full adder & 16 & 4 & 2.4 & 0.61 & 1.2 & 6.14 \\
\hline Oscillator with 3 inverters & 5 & 4 & 8.9 & 0.62 & 1.2 & 1.21 \\
\hline 2 bit carry look-ahead adder & 46 & 6 & 2.4 & 0.62 & 1.2 & 8.65 \\
\hline 2 bit XOR & 8 & 4 & 2.4 & 0.61 & 1.2 & 6.24 \\
\hline 2 bit NOR & 6 & 4 & 1.9 & 0.64 & 1.2 & 4.64 \\
\hline 2 bit NAND & 6 & 4 & 1.9 & 0.64 & 1.2 & 4.86 \\
\hline 2 bit magnitude & 24 & 6 & 2.0 & 0.61 & 1.2 & 10.53 \\
\hline $2-4$ decoder & 16 & 8 & 1.9 & 0.62 & 1.2 & 9.61 \\
\hline Priority 4-2 encoder & 7 & 4 & 1.9 & 0.62 & 1.2 & 5.54 \\
\hline D-latch & 4 & 2 & 2.3 & 0.68 & 1.1 & 3.10 \\
\hline $\mathrm{D}-\mathrm{F} / \mathrm{F}$ & 8 & 4 & 1.9 & 0.65 & 1.1 & 6.90 \\
\hline 2 bit branch & 32 & 12 & 2.2 & 0.66 & 1.1 & 28.6 \\
\hline
\end{tabular}

\subsection{Timing behavior}

We estimate the timing behavior of the benchmark circuits by SPICE simulation and we calculate three parameters for each circuit when the acceptor's lifetime is chosen to be $1 \mathrm{ps}$ : (1) propagation delay, (2) rise time, and (3) fall time. Similar to conventional logic technologies, the propagation delay of a gate defines the time for the output to respond to a change of its inputs. Similarly, rise time and fall time express how fast a signal transits between different levels and they are usually defined as the time between $10 \%$ and $90 \%$ points of output waveforms.

In contrast to CMOS circuits where various inputs and outputs affect the capacitance and therefore timing, the timing behavior of our system is not sensitive to different frequencies of input. As a result, we trace the input and output signals of only one combination of 1-0 or 0-1 transitions for circuits in Table 1, and then use Matlab to process the trace data to extract the three delay parameters shown in this table. From this data we can see that all propagation delay is within 3 ps for all but one, and especially for five of them, is $1.9 \mathrm{ps}$, which is even smaller than a single inverter delay in $22 \mathrm{~nm}$

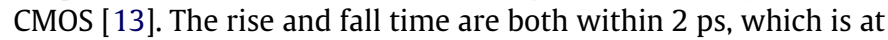
least $10 \times$ smaller than the $25-200$ ps rise and fall times in $22 \mathrm{~nm}$ CMOS with a loading capacitance from $1 \mathrm{fF}$ to $10 \mathrm{pF}$ [16].

To inspect the details of how signals propagate in the system, we draw an amplitude-frequency-time contour plot for a 2 bit multiplexer (Fig. 19(a)) and an amplitude versus time plot of output signals (Fig. 19(b)). From both figures we see that the true and complementary signals alternate, and the times at which output signals are generated are disjoint in steady-state. Furthermore, the Gaussian noise terms are clearly present on both the true and complementary signals in the contour plot.

Fig. 19(a) shows how the dual-rail optical frequency components evolve in time as the inputs to the 2-input multiplexer change. The component near $750 \mathrm{THz}$ is the true form of the function and the component near $650 \mathrm{THz}$ is the complement form. We note that the Gaussian nature of the frequency spread is similar to the spectral spread of a chromophore and that the components are correctly mutually exclusive in steady-state.

In conclusion, all the circuits we have implemented and analyzed are much faster, than $22 \mathrm{~nm}$ CMOS when the lifetime of an accepter is set to be $1 \mathrm{ps}$. When shorter lifetime of an accepter is chosen, the timing behavior of the OLE circuits can be even better with shorter delays. Next, we analyze the power and energy performance of OLE-based logic circuits using a power model derived from the SPICE results presented here.

\subsection{Power dissipation}

We construct a power dissipation model for each component in the optical system and map them to the SPICE model. The minterm 
a

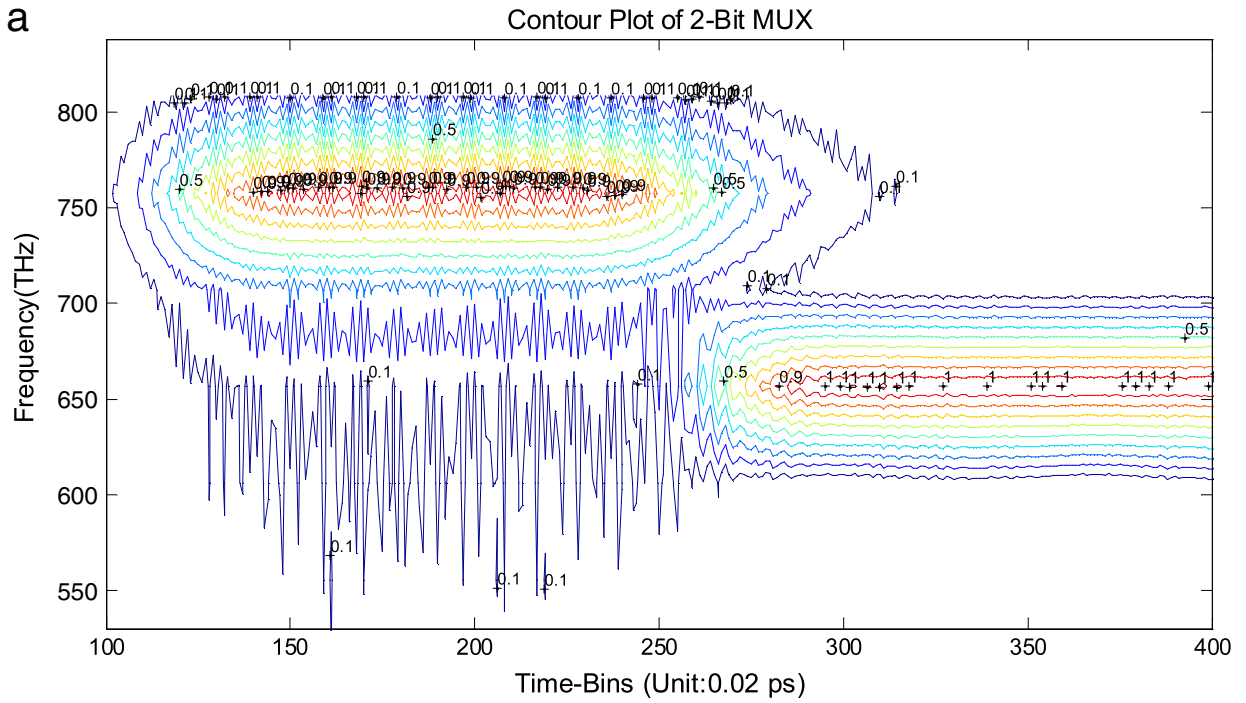

b 2 -Bit MUX Output

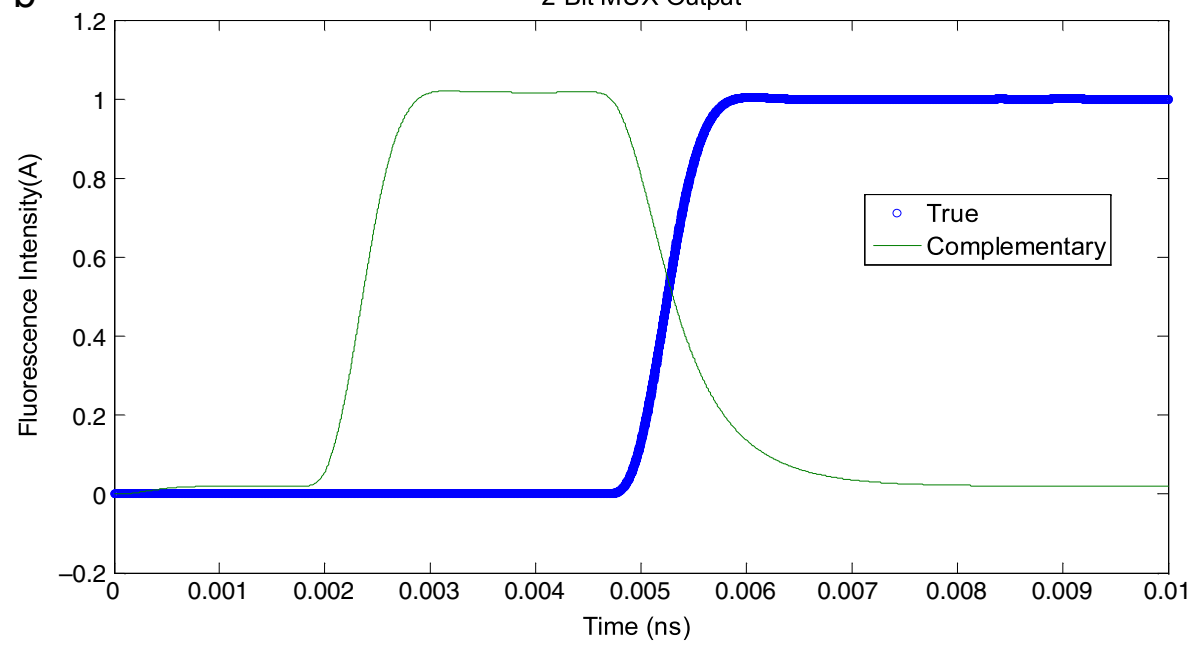

Fig. 19. 2-bit multiplexer simulation results (a) contour graph (b) time domain true and complement frequencies.

power model includes the power dissipation equations of nonradiative RET and the radiative fluorescence process, which are similar to a power model proposed by Pistol et al. [26]. Instead of considering one donor-acceptor pair, the minterms need up to five donors as inputs and one acceptor as the output. The fiber and color filter models attenuate signals at a constant rate which contributes to the power dissipation and is directly calculated.

The power model for quantum dots is shown in Table 3. In this model we consider two optical pumps. One is a gain pump which is used for population inversion of input photons [20]. The gain pump brings the quantum dots to a voltage level which is close to the preset threshold. Then if any photon is absorbed to have the total voltage greater than the threshold, the number of the output photons will be increased as well as the intensity.

The other optical pump is an up-conversion pump which shifts the wavelength of input photons [33,22]. We assume photons are amplified first, and then only $E_{u p}$ fraction of the photons are upconverted. The total power thermalized in the quantum dot layer includes the parts dissipated by two pumps and also energy from the photons that are not up-converted after the gain process due to inefficiencies.

We map the power model to SPICE by using current to represent quantities of photons. The constants we choose define the amount of current for each photon as $1 \mathrm{~A} / \mathrm{ph}$ for convenience of calculation.
Table 3

Power model for the quantum dot layer.

\begin{tabular}{ll}
\hline Term & Definition \\
\hline$G$ & Intensity gain of quantum dots \\
$\alpha_{1}$ & Gain pump utilization efficiency \\
$\alpha_{2}$ & Up-conversion pump utilization \\
& efficiency \\
$k_{g}$ & Gate rate \\
$E_{\text {up }}$ & Efficiency of up-conversion \\
$P_{\text {gain_pump }}=\frac{(G-1) k_{g} h v_{\text {out }}\left(1-\alpha_{1}\right)}{\alpha_{1}}$ & Power thermalized for gain pump \\
$P_{\text {up_pump }}=\frac{G k_{\text {out }} h\left(v_{i}-E_{\text {up }}\left(1-\alpha_{2}\right)\right.}{\alpha_{2}}$ & Power thermalized for \\
$P_{\text {non_up }}=G k_{g} h v_{\text {out }}\left(1-E_{\text {up }}\right)$ & up-conversion pump \\
$P_{q d}=P_{\text {gain_pump }}+P_{\text {up_pump }}+P_{\text {non_up }}$ & Power thermalized for \\
& non-up-converted dyes \\
& power thermalized for quantum \\
\hline
\end{tabular}

We assume at least one photon for an input or output is needed to make the circuit work, and the total number of input photons is the same as that of output photons. From this we can estimate the energy dissipation (per transition) for all the circuits shown in Table 2 by considering the worst case which defines the upper bound of the energy dissipation. The absolute values of energy consumption per transition are shown in the rightmost column in Table 2. 
We compare the energy/transition of several basic circuits like the 2-input NAND, NOR and XOR gates with power-delay products (PDP) of the same circuits in $32 \mathrm{~nm}$ CMOS [7] and find the RET logic presents a significant advantage. The simulated energy dissipation of the RET logic circuits are at least an order of magnitude less than the $32 \mathrm{~nm}$ CMOS technology and even the 2-bit branch predictor has about $10 \times$ less energy than a simple two input CMOS NOR gate. If we compare the RET logic with the $22 \mathrm{~nm}$ tri-gates from Intel, which claim to have $50 \%$ power reduction with the same performance [6], the RET logic has at least $5 \times$ better PDP. If we compare it with the $14 \mathrm{~nm}$ FinFETs which potentially offer up to $50 \%$ performance improvement or $50 \%$ power reduction compared with a $28 \mathrm{~nm}$ process [12], the PDP of the RET logic is still $2.5 \times$ better. Moreover, with at least two orders of magnitude better area density than the state-of-the-art CMOS technology as analyzed in Section 2.2.3, the RET-logic based technology shows an even greater advantage than CMOS.

\section{Conclusions and future work}

The Optical Logic Element (OLE) we present is a layered structure similar to a five-input lookup table (5-LUT) and can be fabricated by self-assembly. Unlike traditional CMOS, an OLE uses wavelength division multiplexing for its inputs and output. Conventional optical fibers are used in a platform for studying the OLE structure and in the future will serve as wires to interconnect multiple OLEs into larger systems.

To better understand the characteristics of the system and to be useful as a guide for future experimental demonstration, a SPICE model for each component including a mapped power model is built from first principles and verified with experimental results. Various combinational and sequential circuits are designed and simulated to estimate their timing and power behavior. The simulation results suggest that the propagation delay, rise and fall times in this system are much faster than the $22 \mathrm{~nm}$ CMOS technology and that the system is at least $2.5 \times$ more energy efficient as well. Moreover, the area density of the OLE-based technology is at least two orders of magnitude better than the state-of-the-art CMOS technology.

Future work will focus on the experimental demonstration of integrated OLEs and the study of various methods to improve the longevity of the chromophores within. Beyond molecular techniques to solve these problems, we will also investigate various fault-tolerant circuit design approaches as they apply to building larger systems from OLEs.

\section{References}

[1] M. Achermann, M. Petruska, S. Kos, D. Smith, D. Koleske, V. Klimov, Energytransfer pumping of semiconductor nanocrystals using an epitaxial quantum well, Nature 429 (6992) (2004) 642-646.

[2] M. Anni, L. Manna, R. Cingolani, D. Valerini, A. Creti, M. Lomascolo, Förster energy transfer from blue-emitting polymers to colloidal CdSe/ZnS core shell quantum dots, Appl. Phys. Lett. 85 (18) (2004) 4169-4171.

[3] C. Beetz, R. Boerstler, J. Steinbeck, B. Lemieux, D. Winn, Silicon-micromachined microchannel plates, Nucl. Instrum. Methods Phys. Res. A 442 (1) (2000) 443-451.

[4] A. Biberman, N. Sherwood-Droz, X. Zhu, K. Preston, G. Hendry, J.S. Levy, J. Chan, H. Wang, M. Lipson, K. Bergman, Photonic network-on-chip architecture using 3d integration, in: Proc. of SPIE, vol. 7942, 2011, p. 79420M-1.

[5] A. Biswas, P. Basu, Equivalent circuit models of quantum cascade lasers for SPICE simulation of steady state and dynamic responses, J. Opt. A: Pure Appl. Opt. 9 (1) (2006) 26.

[6] M. Bohr, K. Mistry, Intels revolutionary $22 \mathrm{~nm}$ transistor technology, Rob Willoner at Innovation.

[7] G. Cho, Y. Kim, F. Lombardi, Assessment of CNTFET based circuit performance and robustness to pvt variations, in: Circuits and Systems, 2009, 52nd IEEE International Midwest Symposium on MWSCAS'09, IEEE, 2009, pp. 1106-1109.

[8] G. Drobychev, A. Barysevich, K. Delendik, A. Karneyeu, P. Nédélec, D. Sillou, O. Voitik, Development of micro-channel plates on a basis of aluminum oxide, Nucl. Instrum. Methods Phys. Res. A 567 (1) (2006) 290-293.
[9] C. Dwyer, V. Johri, M. Cheung, J. Patwardhan, A. Lebeck, D. Sorin, Design tools for a DNA-guided self-assembling carbon nanotube technology, Nanotechnology 15 (9) (2004) 1240.

[10] J. Foresi, P. Villeneuve, J. Ferrera, E. Thoen, G. Steinmeyer, S. Fan, J. Joannopoulos, L. Kimerling, H. Smith, E. Ippen, Photonic-bandgap microcavities in optical waveguides, Nature 390 (6656) (1997) 143-145.

[11] J. Glesener, J. Estrera, Two micron pore size mcp-based image intensifiers, in: OPTO, International Society for Optics and Photonics, 2010 pp. 759814-759814.

[12] C.-P. Hsu, $16 \mathrm{~nm} / 14 \mathrm{~nm}$ FinfETs: enabling the new electronics frontier http://electronicdesign.com/digital-ics/16nm14nm-finfets-enabling-newelectronics-frontier.

[13] Z. Huang, A. Kurokawa, M. Hashimoto, T. Sato, M. Jiang, Y. Inoue, Modeling the overshooting effect for cmos inverter delay analysis in nanometer technologies, IEEE Trans. Comput.-Aided Des. Integr. Circuits Syst. 29 (2) (2010) 250-260.

[14] Hamamatsu, http://sales.hamamatsu.com/assets/pdf/catsandguides/ii_tii0004e02.pdf.

[15] A. Joshi, C. Batten, Y. Kwon, S. Beamer, I. Shamim, K. Asanovic, V. Stojanovic, Silicon-photonic close networks for global on-chip communication, in: Proceedings of the 2009 3rd ACM/IEEE International Symposium on Networkson-Chip, IEEE Computer Society, 2009, pp. 124-133.

[16] D. Kim, S. Kim, S. Lim, Impact of nano-scale through-silicon vias on the quality of today and future 3d IC designs, in: Proceedings of the System Level Interconnect Prediction Workshop, IEEE Press, 2011, p. 1.

[17] N. Kirman, M. Kirman, R. Dokania, J. Martinez, A. Apsel, M. Watkins, D. Albonesi, Leveraging optical technology in future bus-based chip multiprocessors, in: Proceedings of the 39th Annual IEEE/ACM International Symposium on Microarchitecture, IEEE Computer Society, 2006, pp. 492-503.

[18] V. Klimov, Nanocrystal Quantum Dots, CRC Press Boca Raton, FL, 2010.

[19] V. Klimov, S. Ivanov, J. Nanda, M. Achermann, I. Bezel, J. McGuire, A. Piryatinski, Single-exciton optical gain in semiconductor nanocrystals, Nature 447 (7143) (2007) 441-446

[20] V. Klimov, A. Mikhailovsky, S. Xu, A. Malko, J. Hollingsworth, C. Leatherdale H. Eisler, M. Bawendi, Optical gain and stimulated emission in nanocrystal quantum dots, Science 290 (5490) (2000) 314-317.

[21] J.R. Lakowicz, Principles of Fluorescence Spectroscopy, Springer, 2009.

[22] J. Ouyang, J. Ripmeester, X. Wu, D. Kingston, K. Yu, A. Joly, W. Chen, Upconversion luminescence of colloidal $\mathrm{CdS}$ and $\mathrm{ZnCdS}$ semiconductor quantum dots, J. Phys. Chem. C 111 (44) (2007) 16261-16266.

[23] Y. Pan, P. Kumar, J. Kim, G. Memik, Y. Zhang, A. Choudhary, Firefly: illuminating future network-on-chip with nanophotonics, in: Proceedings of the International Symposium on Computer Architecture, 2009.

[24] I. Petkova, G. Dobrikov, N. Banerji, G. Duvanel, R. Perez, V. Dimitrov, P. Nikolov, E. Vauthey, Tuning the excited-state dynamics of GFP-inspired imidazolone derivatives, J. Phys. Chem. A 114 (1) (2009) 10-20.

[25] C. Pistol, W. Chongchitmate, C. Dwyer, A. Lebeck, Architectural impnlications of nanoscale-integrated sensing and computing, IEEE Micro 30 (1) (2010) $110-120$.

[26] C. Pistol, C. Dwyer, A. Lebeck, Nanoscale optical computing using resonance energy transfer logic, IEEE Micro 28 (6) (2008) 7-18.

[27] C. Pistol, V. Mao, V. Thusu, A. Lebeck, C. Dwyer, Encoded multichromophore response for simultaneous label-free detection, Small 6 (7) (2010) 843-850.

[28] A.P. de Silva, Molecular logic gate arrays, Chem. Asian J. 6 (3) (2011) 750.

[29] B. Valeur, Molecular fluorescence: principles and applications.

[30] T. Vandeweyer, J. De Backer, J. Versluijs, V. Truffert, S. Verhaegen, M. Ercken, M. Dusa, Patterning challenges in setting up a $16 \mathrm{~nm}$ node 6T-SRAM device using euv lithography, in: Proc. SPIE, vol. 7969, 2011, p. 79691K.

[31] D. Vantrease, R. Schreiber, M. Monchiero, M. McLaren, N.P. Jouppi, M. Fiorentino, A. Davis, N. Binkert, R.G. Beausoleil, J.H. Ahn, Corona: system implications of emerging nanophotonic technology, in: Proceedings of the 35th Annual International Symposium on Computer Architecture, IEEE Computer Society, Washington, DC, USA, 2008, pp. 153-164.

[32] C. Wang, L. Lin, Nanoscale waveguiding methods, Nanoscale Res. Lett. 2 (5) (2007) 219-229.

[33] X. Wang, W. Yu, J. Zhang, J. Aldana, X. Peng, M. Xiao, Photoluminescence upconversion in colloidal CdTe quantum dots, Phys. Rev. B 68 (12) (2003) 125318.

[34] F.Zhang, R. Tang, Y. Kim, Set-based nano-circuit simulation and design method using hSPICE, Microelectron. J. 36 (8) (2005) 741-748.

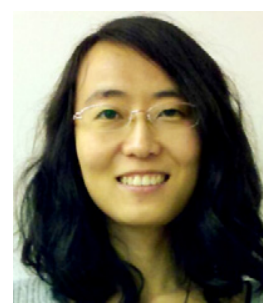

Jun Pang received her B.S. in information engineering from Zhejiang University in China, and her M.S in signal and information processing from Chinese Academy of Science. She is currently a Ph.D. candidate at the Department of Computer Science, Duke University. Her research interests include computer architecture for emerging nanotechnologies, nanophotonic Network-onChip, and design and simulation of digital circuits. 


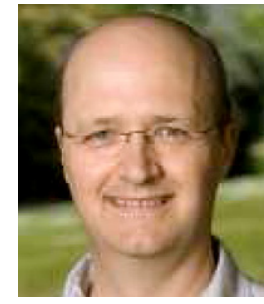

Alvin R. Lebeck received the B.S. degree in electrical and computer engineering, and the M.S. and Ph.D. degrees in computer science from the University of Wisconsin Madison. He is an professor of computer science and of electrical and computer engineering at Duke University. His research interests include architectures for emerging nanotechnologies, high performance microarchitectures, hardware and software techniques for improved memory hierarchy performance, multiprocessor systems, and energy efficient computing. He received the best paper award at the 31st IEEE/ACM International Symposium on Microarchitecture. He is the recipient of a 1997 US National Science Foundation (NSF) CAREER Award, has received funding from the NSF, DARPA, Intel, Compaq, Microsoft, IBM, and is a member of ACM and a senior member of the IEEE and the IEEE Computer Society.

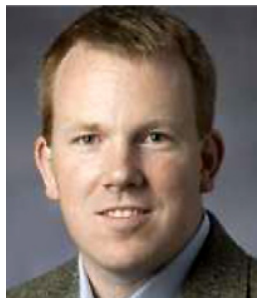

Christopher Dwyer received his B.S. in computer engineering from the Pennsylvania State University in 1998, and his M.S. and Ph.D. in computer science from the University of North Carolina at Chapel Hill in 2000 and 2003, respectively. He is currently an Associate Professor at Duke University having joined the Department of Electrical and Computer Engineering in mid-2004, and is a co-founder and Senior Research Scientist at Parabon NanoLabs, Inc., an applied DNA nanotechnology company. He was awarded a Young Investigator Award from the Army Research Office in 2008 for which he received the Presidential Early Career Award for Scientists and Engineers (PECASE). Dwyer is a Senior Member of the ACM and IEEE and was elected a Kavli Fellow by the National Academy of Sciences in 2011. His areas of research include DNA self-assembly and applications that expand the computational domain with a focus on device-to-systems design, evaluation, and synthesis. 